The Astrophysical Journal, 670:1214-1224, 2007 December 1

(C) 2007. The American Astronomical Society. All rights reserved. Printed in U.S.A.

\title{
DYNAMICAL MASSES FOR PRE-MAIN-SEQUENCE STARS: A PRELIMINARY PHYSICAL ORBIT FOR V773 TAU A
}

\author{
Andrew F. Boden, ${ }^{1,2}$ Guillermo Torres, ${ }^{3}$ Anneila I. Sargent, ${ }^{4}$ Rachel L. Akeson, ${ }^{1}$ John M. Carpenter, ${ }^{4}$ \\ David A. Boboltz, ${ }^{5}$ Maria Massi, ${ }^{6}$ Andrea M. Ghez, ${ }^{7}$ David W. Latham, ${ }^{3}$ \\ Kenneth J. Johnston, ${ }^{5}$ Karl M. Menten, ${ }^{6}$ and Eduardo Ros ${ }^{6}$ \\ Received 2007 March 31; accepted 2007 June 15
}

\begin{abstract}
We report on interferometric and radial velocity observations of the double-lined 51 day period binary (A) component of the quadruple pre-main-sequence (PMS) system V773 Tau. With these observations we have estimated preliminary visual and physical orbits of the V773 Tau A subsystem. Among other parameters, our orbit model includes an inclination of $66.0^{\circ} \pm 2.4^{\circ}$ and allows us to infer the component dynamical masses and system distance. In particular, we find component masses of $1.54 \pm 0.14$ and $1.332 \pm 0.097 M_{\odot}$ for the Aa (primary) and $\mathrm{Ab}$ (secondary) components, respectively. Our modeling of the subsystem component spectral energy distributions finds temperatures and luminosities consistent with previous studies and, coupled with the component mass estimates, allows for comparison with PMS stellar models in the intermediate-mass range. We compare V773 Tau A component properties with several popular solar composition models for intermediate-mass PMS stars. All models predict masses consistent to within $2 \sigma$ of the dynamically determined values, although some models predict values that are more consistent than others.
\end{abstract}

Subject headings: binaries: spectroscopic — stars: fundamental parameters — stars: individual (V773 Tau) — stars: pre-main-sequence

\section{INTRODUCTION}

Accurate determinations of the physical properties of stars (mass, radius, temperature, luminosity, elemental abundances, rotation, etc.) provide fundamental tests of stellar structure and evolution models. Among the areas where our understanding of stellar structure is most uncertain is in pre-main-sequence (PMS) stars, particularly for low-mass systems (see Palla \& Stahler 2001; Hillenbrand \& White 2004; Mathieu et al. 2007 and references for summaries). Previously we have reported results from our program of studying PMS binary systems with the intent of establishing dynamical mass and luminosity constraints (Boden et al. 2005b). Here we report on observations of the double-lined spectroscopic binary V773 Tau A, part of the PMS quadruple system V773 Tau.

V773 Tau (HDE 283447, HBC 367) is among the most interesting and well-studied systems in the Taurus-Auriga star-forming region. V773 Tau was first identified as a T Tauri star by Rydgren et al. (1976) based on $\mathrm{H} \alpha$ and $\mathrm{Ca}$ II $\mathrm{H}$ and $\mathrm{K}$ emission, high lithium abundance, photometric variability, and K2 spectral type. The object presented an enigmatic mixture of classical (CTTS) and weak-lined T Tauri (WTTS) characteristics until it became apparent that there are multiple components; V773 Tau was resolved as a visual binary independently by Ghez et al. (1993) and Leinert et al. (1993) (with components designated here as

\footnotetext{
1 Michelson Science Center, California Institute of Technology, Pasadena CA 91125; bode@ipac.caltech.edu.

2 Department of Physics and Astronomy, Georgia State University, Atlanta, GA 30303.

3 Harvard-Smithsonian Center for Astrophysics, Cambridge, MA 02138.

4 Division of Physics, Math, and Astronomy, California Institute of Technology, MS 105-24, Pasadena, CA 91125.

5 US Naval Observatory, Washington, DC 20392.

6 Max-Planck-Institut für Radioastronomie, D-53121 Bonn, Germany.

7 Division of Astronomy and Astrophysics, UCLA, Los Angeles, CA 900951562 .
}

A and B). Martín et al. (1994) suggested, and Welty (1995, hereafter W95) established, A as a short-period (51 day) double-lined spectroscopic binary. In high angular resolution studies Duchene et al. (2003, hereafter D03) and Woitas (2003) independently identified an additional, "infrared" component in the system (herein designated C; note that D03 use an alternate component notation), making V773 Tau an apparent compact quadruple system with four stars within roughly $100 \mathrm{AU} .{ }^{8}$ Using spatially resolved photometry, D03 find the A, B, and C components to have distinctly different spectral energy distributions (SEDs). D03 conclude that V773 Tau contains stars at very different PMS evolutionary states: the optically brighter A stars are WTTS in character, while the circumstellar material inferred from the significant IR excess is associated with the $\mathrm{B}$ and $\mathrm{C}$ components. D03 and Tamazian et al. (2002) have estimated the V773 Tau A-B visual orbit from relative astrometry.

Further, the V773 Tau A subsystem is a bright, polarized, and highly variable radio source. Kutner et al. (1986) discovered radio continuum emission from the system, appearing as the most luminous Taurus-Auriga T Tauri star in the survey by O'Neal et al. (1990). Feigelson et al. (1994) published a comprehensive multiwavelength study of the V773 Tau system, observing both polarized and flaring radio emission. W95 argued that both A components are chromospherically active based on variations in their optical spectrum and estimates of relatively rapid rotation ( $v \sin i \sim 40 \mathrm{~km} \mathrm{~s}^{-1}$ ); this point was confirmed by Phillips et al.

\footnotetext{
8 There are at least three different component notations used in describing the V773 Tau system. Herein we have described the 51 day SB2 subsystem as "A," with stellar components $\mathrm{Aa}$ and $\mathrm{Ab}$; this is consistent with nomenclature used in Massi et al. (2006) and Woitas (2003). Conversely, D03 refer to the 51 day SB2 subsystem as "AB." Finally, based on arguments made by Hartigan et al. (1994) on the "likely" association of V773 Tau and FM Tau, the Washington Double Star Catalog (Mason et al. 2001) describes the four-star complex as "A" (with FM Tau as "B") and the 51 day SB2 as "Aa" with components "Aa1" and "Aa2."
} 
TABLE 1

V773 Tau A Ki $V^{2}$ Data Set, "Hybrid" Orbit Model Predictions, and Residuals

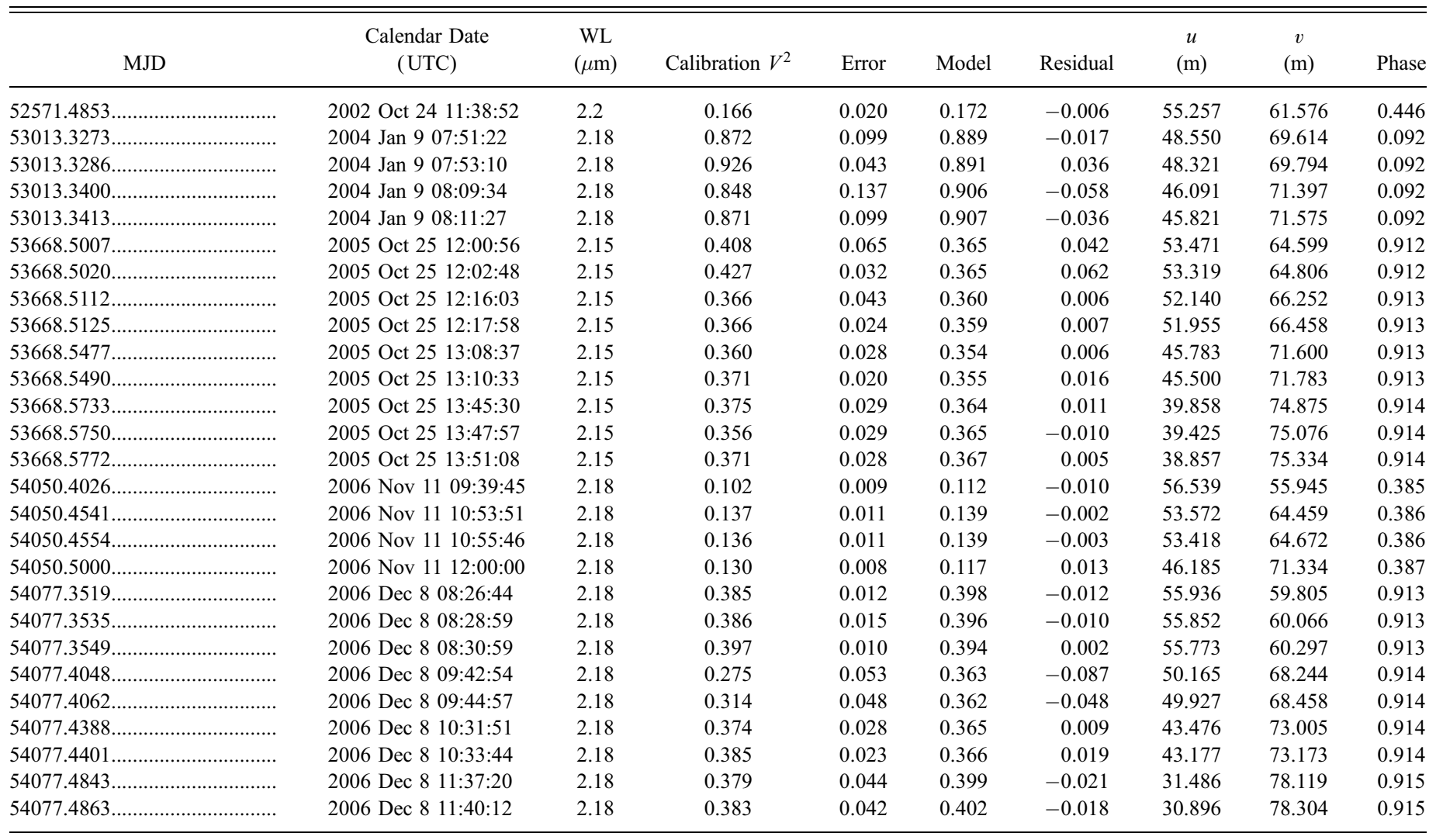

Note.--Residuals are quoted as data minus model.

(1996, hereafter P96), who resolved the radio emission into a clear binary morphology apparently corresponding to the two A components. Massi et al. (2002) identified an increase in flare activity around A periastron, and Massi et al. (2006) studied one such flare in detail; more frequent and powerful radio flaring at periastron is apparently due to interaction of component magnetic fields while in close proximity. Finally, Lestrade et al. (1999, hereafter L99) used Very Long Baseline Interferometry (VLBI) astrometry to measure the trigonometric parallax for V773 Tau A as $6.74 \pm 0.25$ mas, corresponding to a system distance of $148.4 \pm 5.5 \mathrm{pc}$.

Here we report on observations of the V773 Tau A subsystem made with the Keck Interferometer (KI; see Colavita et al. 2003), the Very Long Baseline Array (VLBA; see Napier et al. 1994; Zensus et al. 1995), and spectroscopic radial velocities (RVs) from the Harvard-Smithsonian Center for Astrophysics (CfA) "Digital Speedometers" (Latham 1992). These observations resolve V773 Tau A and allow us to estimate the visual and physical (i.e., three-dimensional) orbits and to determine the component dynamical masses and system distance. We describe these orbit models as preliminary because they weakly depend on ongoing observations and modeling of the larger V773 Tau system. Combining derived dynamical masses and system distance with component radiometric modeling, we estimate component temperatures and luminosities, allowing us to make direct comparisons with a number of popular solar composition PMS stellar models.

\section{OBSERVATIONS AND ORBITAL SOLUTION}

KI observations.-The KI interferometric observable used for these measurements is the fringe contrast or visibility (squared,
$V^{2}$ ) of an observed brightness distribution on the sky. Orbital analysis methods for such wide-band $V^{2}$ observations are discussed in Boden et al. (2000) and not repeated here. V773 Tau A was observed by $\mathrm{KI}$ in $K$ band $(\lambda \sim 2.2 \mu \mathrm{m})$ on five nights between 2002 October 24 and 2006 December 8, a data set spanning roughly 4 yr and 30 orbital periods. V773 Tau A and calibration objects were typically observed multiple times during each of these nights, and each observation (scan) was approximately $130 \mathrm{~s}$ long. For each interferometric scan we computed a mean (incoherent wide band; Colavita 1999; Colavita et al. 2003) $V^{2}$ value from the scan data, and the error in the $V^{2}$ estimate is inferred from the rms internal scatter. For this analysis we have used HD 27741 (G0 V) and HD 28483 (F6 V) as calibration objects. Calibrating our interferometric data with respect to these objects results in 27 calibrated visibility scans on V773 Tau A over the five epochs. Because the AO-corrected Keck Telescopes separately resolve the V773 Tau A-B-C complex (D03) and the $\mathrm{KI}$ beam combiner is fed by single-mode fiber, no significant light from the $\mathrm{B}$ or $\mathrm{C}$ components enters the fringe camera while observing A (the $K$-band projected FWHM size of the KI singlemode fiber is 50 mas, so the $\mathrm{B}$ component is approximately five $e$-folding lengths away from A at the epoch of our observations; Colavita et al. 2003; D03). Consequently, no special provisions for extra light are necessary in processing KI observations of V773 Tau A.

Our KI $V^{2}$ observations are summarized in Table 1. The $V^{2}$ data set is depicted in Figure 1, along with $V^{2}$ predictions from our "Hybrid" orbit model (discussed below).

VLBI observations. - P96 observed V773 Tau A with the VLBA and, with similar spatial frequencies as our KI observations $(\sim 50 \mathrm{M} \lambda)$, resolved the typical 2 mas separation between the 


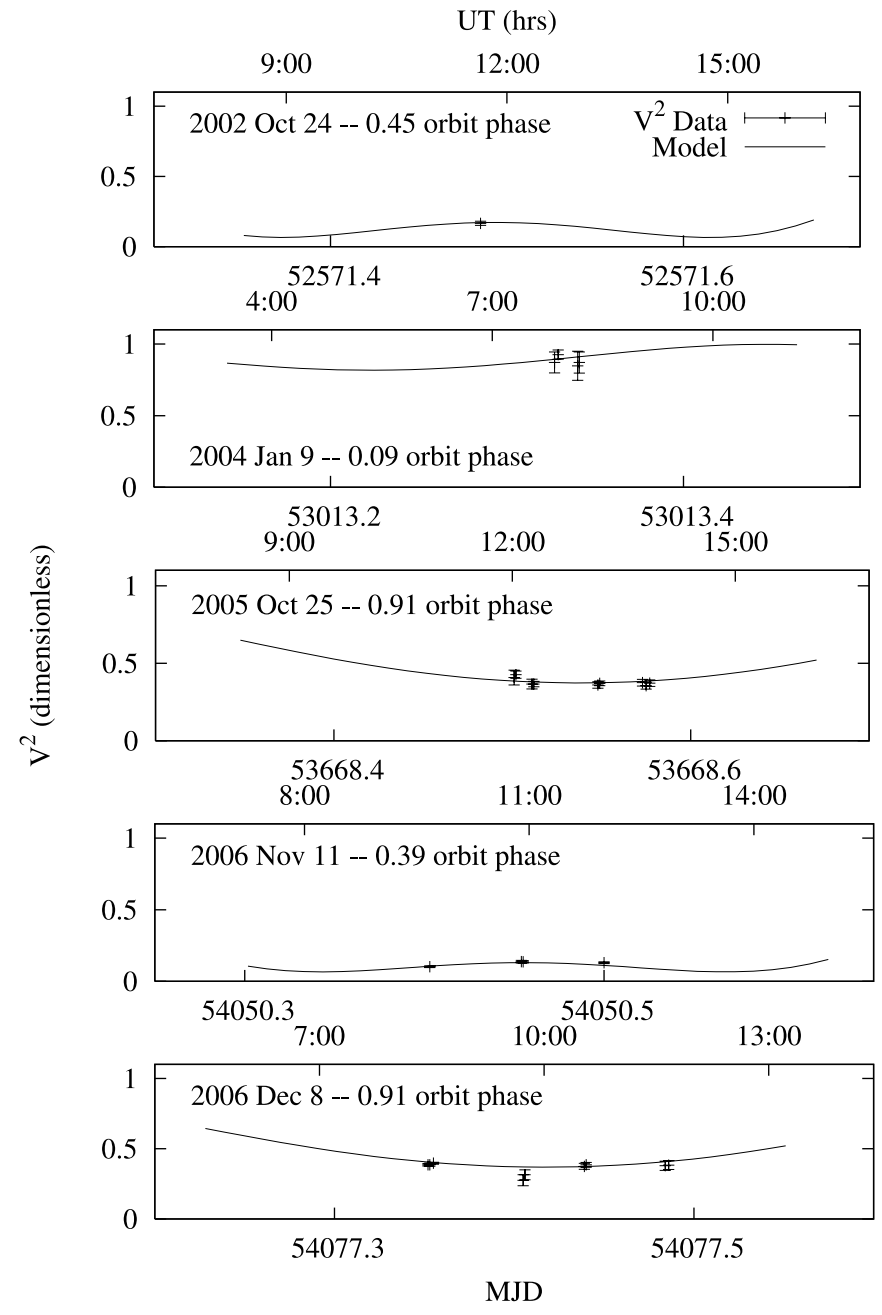

FIG. 1.-KI data/model comparisons for V773 Tau A $V^{2}$ observations. Here we give comparisons for our five epochs of $\mathrm{KI} V^{2}$ observations and $V^{2}$ predictions from our Hybrid orbital model ( Table 4). In each case the data and model are shown.

A components. Figure 6 of P96 depicts a VLBA contour map of V773 Tau A at a particular epoch (1992 September 11) and clearly shows a binary source structure. P96 interpreted this binary morphology as radio flare emission from the two subsystem components. P96 argued that the radio emission is coincident with the stars (to within a "few" stellar diameters), so a component separation interpreted as at-epoch relative astrometry was estimated by $\mathrm{P} 96$.

A team led by one of us (M. M.) has studied the physics of V773 Tau A radio flares. In addition to previous results (e.g., Massi et al. 2002, 2006), V773 Tau A was observed using the VLBA in coordination with the Effelsberg $100 \mathrm{~m}$ radio telescope (herein VLBA+EB). These $8.4 \mathrm{GHz}$ (X band) observations were conducted around apastron on 7 consecutive days (2004 March 11-17 inclusive). As in the case of P96, analysis of these observations revealed what appears to be both A components simultaneously exhibiting radio flares in most of these observations. However, some of these "double-flare" observations show complex emission morphologies, complicating estimation of the binary relative separation. Reviewing the maps from these seven epochs, we decided to use astrometry from observations where there was significant (i.e., that the peak emission was greater than $6 \sigma$ above the rms noise level) emission from both components and the component structures were largely unresolved compared to the VLBA synthetic beam. These criteria resulted in our retaining three of the seven 2004 epochs for orbital analysis purposes; the astrometry resulting from these observations is summarized in Table 2. A sample image constructed from one of the three astrometric epochs (2004 March 15) is shown in Figure 2. The astrometric results of both the P96 and these VLBI observations are depicted in Figure 3; these observations agree well with our orbit model for V773 Tau A described below. The radio morphologies seen in the full VLBI+EB data set will be analyzed in a forthcoming publication (Massi et al. 2007).

RVobservations.-W95 established V773 Tau A as a doublelined spectroscopic binary and published an orbital solution. RV monitoring of V773 Tau with the CfA Digital Speedometers (Latham 1992) was carried out at the Oak Ridge Observatory (Harvard, Massachusetts), the F. L. Whipple Observatory (Mount Hopkins, Arizona), and the MMT Observatory (also on Mount Hopkins, Arizona). The nearly identical instruments at these facilities record a single echelle order and provide a wavelength coverage of $45 \AA$ centered at $5187 \AA$, with a resolving power of $\lambda / \Delta \lambda \approx 35,000$. Wavelength calibration was achieved in the usual manner through exposures of a Th-Ar lamp taken before and after each science exposure. The velocity zero point was monitored by means of exposures of the dusk and dawn sky, and small run-to-run corrections were applied to the velocities derived below as described by Stefanik et al. (1999).

Our spectroscopic observations of V773 Tau began roughly at the same time as the W95 data set and have continued to the present. In total we have collected 52 spectra spanning the period 1991 September 25 to 2007 January 7, with signal-to-noise ratios $(\mathrm{S} / \mathrm{Ns})$ of $16-35$ per resolution element of $8.5 \mathrm{~km} \mathrm{~s}^{-1}$. The RVs derived from these observations are summarized in Table 3. RVs were derived using the two-dimensional cross-correlation technique TODCOR (Zucker \& Mazeh 1994), with templates adopted from a large library of calculated spectra based on Kurucz model atmospheres (see also Nordström et al. 1994; Latham et al. 2002). The best-matching templates were chosen by running large grids of cross-correlations as described by Torres et al. (2003), which also yielded estimates of some of the stellar parameters. For an assumed Taurus solar metallicity (see Padgett 1996) and surface gravities appropriate for these stars $(\log g \approx 4.0$; see

TABLE 2

V773 Tau A Vlbi Astrometry Data Set, "Hybrid" Orbit Model Predictions, and Residuals

\begin{tabular}{|c|c|c|c|c|c|c|c|c|c|c|c|}
\hline MJD & $\begin{array}{c}\text { Calendar } \\
\text { Date }\end{array}$ & $\begin{array}{c}\text { Separation } \\
\text { R.A. }\end{array}$ & $\begin{array}{l}\text { Separation } \\
\text { Decl. }\end{array}$ & $\begin{array}{l}\text { Error } \\
\text { R.A. }\end{array}$ & $\begin{array}{l}\text { Error } \\
\text { Decl. }\end{array}$ & $\begin{array}{l}\text { Model } \\
\text { R.A. }\end{array}$ & $\begin{array}{l}\text { Model } \\
\text { Decl. }\end{array}$ & $\begin{array}{c}\text { Residual } \\
\text { R.A. }\end{array}$ & $\begin{array}{c}\text { Residual } \\
\text { Decl. }\end{array}$ & Phase & Comment \\
\hline $48876.500 \ldots \ldots \ldots \ldots$ & 1992 Sep 11 & -0.85 & 0.76 & 0.2 & 0.2 & -0.631 & 0.817 & -0.217 & -0.061 & 0.143 & P96 \\
\hline $53079.840 \ldots \ldots \ldots \ldots$ & 2004 Mar 15 & 2.78 & 2.04 & 0.2 & 0.2 & 2.742 & 1.869 & 0.037 & 0.170 & 0.393 & BM198 epoch E \\
\hline $53080.840 \ldots \ldots \ldots \ldots$ & 2004 Mar 16 & 3.18 & 1.56 & 0.2 & 0.2 & 2.885 & 1.828 & 0.292 & -0.272 & 0.413 & BM198 epoch F \\
\hline
\end{tabular}

NotE.-Residuals are quoted as data minus model; astrometric quantities are in mas. 


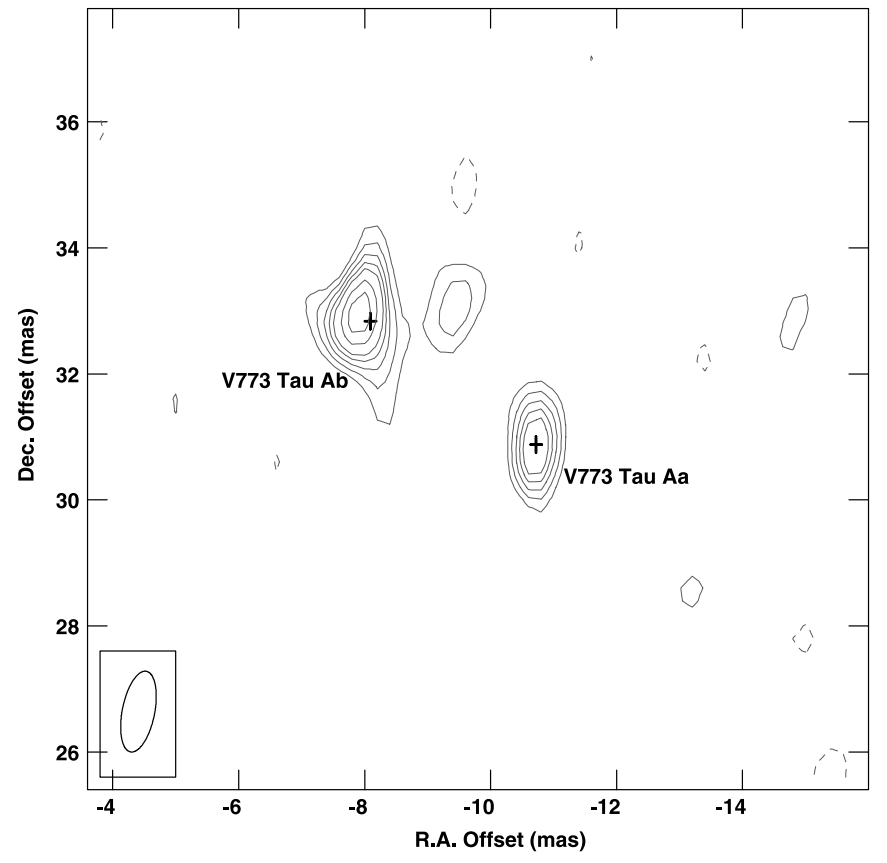

FIG. 2.-Sample VLBA map from 2004 March 15. Here we give a contour map of the total intensity continuum emission toward V773 Tau A from VLBA+ EB observations of 2004 March 15. Contours are at $-3,3,4.5,6,7.5,9,12$, and $15 \sigma$ of the rms noise in the image $\left(0.1 \mathrm{mJy}^{-1}\right.$ beam $\left.^{-1}\right)$. The FWHM beam size (lower left) is $1.36 \times 0.50$ mas at a position angle of $-11.6^{\circ}$. The peak flux density in the image is $0.55 \mathrm{mJy}^{\text {beam }}{ }^{-1}$. V773 Tau A is offset in these data and image as a calibrator ( $0403+2600$, not shown) served as the phase center. Thick plus signs are rendered at the predicted stellar separation based on our " $V{ }^{2}$ and RV" orbit from Table 4 (i.e., no radio data included in the orbit modeling), registered to the center of the apparent primary position. The sizes of the plus signs indicate the uncertainty in the separation estimate ( $\sim 0.1$ mas per axis).

Table 5 below), we derived effective temperatures of $4900 \pm 150$ and $4740 \pm 200 \mathrm{~K}$ for $\mathrm{Aa}$ and $\mathrm{Ab}$, respectively. The adopted errors are conservative estimates to account for the abundance uncertainty. Formal errors are $50 \mathrm{~K}$ smaller and are based on the scatter from the different spectra. Using the same methods, we estimated the projected rotational velocity for the primary star to be $v \sin i=38 \pm 4 \mathrm{~km} \mathrm{~s}^{-1}$, in good agreement with the determination by W95 (41.4 $\mathrm{km} \mathrm{s}^{-1}$; no uncertainty reported). We were not able to determine the $v \sin i$ for the fainter secondary, possibly due to the relatively low $\mathrm{S} / \mathrm{Ns}$ of our spectra. The templates for our RV determinations were selected to have temperatures of 5000 and $4750 \mathrm{~K}$ (the nearest values in our template library) for $\mathrm{Aa}$ and $\mathrm{Ab}$, respectively, and rotational velocities of $40 \mathrm{~km} \mathrm{~s}^{-1}$ for both stars. The light ratio between the secondary and the primary was determined with TODCOR by leaving it as an additional free parameter during the velocity determinations, as described by Zucker \& Mazeh (1994). We obtained $l_{\mathrm{Ab}} / l_{\mathrm{Aa}}=0.37 \pm 0.03$ at a mean wavelength of $5187 \AA$.

The presence of two additional spatially unresolved components in the V773 Tau system may in principle affect both our determination of the stellar parameters of $\mathrm{Aa}$ and $\mathrm{Ab}$ and their RVs. While the infrared companion V773 Tau C (D03; Woitas 2003) is much too faint to cause any contamination, V773 Tau B is estimated to be $\sim 1.7$ mag fainter than the spectroscopic binary in the optical (D03) and could possibly have an effect. We attempted to detect the lines of this component in our spectra using an extension of TODCOR to three dimensions (see Zucker et al. 1995) but were unsuccessful. On this basis we conclude that it does not significantly affect our results above. However, its presence does complicate the analysis of the RV data for the
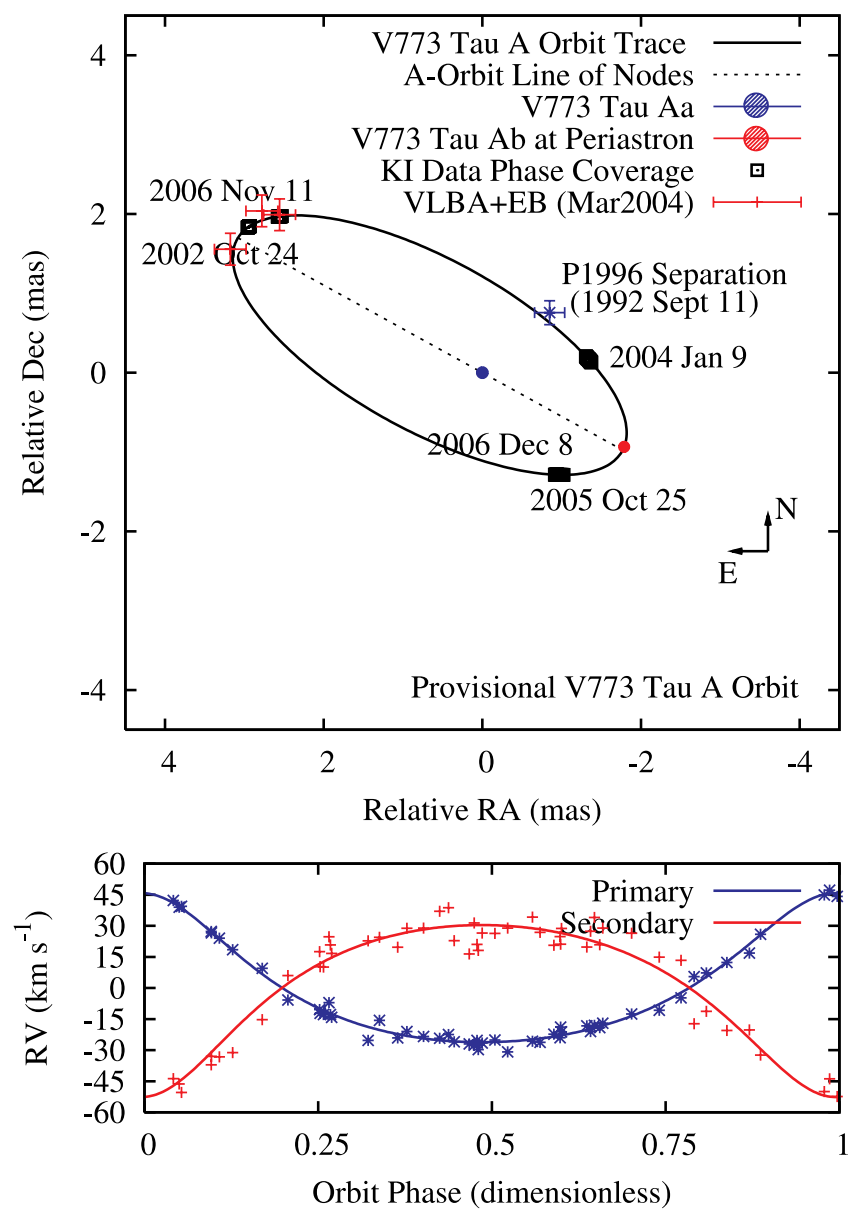

FIG. 3.- Orbit of V773 Tau A as derived in our Hybrid solution (Table 4). Top: Relative visual orbit model of V773 Tau A, with the primary and secondary objects rendered at $T_{0}$ (periastron). The specific epochs where we have KI $V^{2}$ phase coverage are indicated on the relative orbit (they are not separation vector estimates), and the VLBI separations from P96 (in blue) and this work (in red) are also shown. Component diameter values are estimated and rendered to scale. Bottom: Double-lined RV orbit model and data described here.

spectroscopic binary in that the motion in the A-B orbit needs to be taken into account. We discuss this further below and in a forthcoming publication (G. Torres et al. 2007, in preparation).

Orbital solution.-We have combined the astrometric (KI, P96, VLBA+EB) and RV data described above to estimate the visual and physical (i.e., three-dimensional) orbits for V773 Tau A. In particular, methods for modeling the wide-band visibility data used in this analysis are described in Boden et al. (2000) and not repeated here. Our "Hybrid" orbital solution ( Table 4) is depicted in Figure 3. The top panel depicts the relative visual orbit model, with the primary (Aa) component rendered at the origin and the secondary $(\mathrm{Ab})$ component rendered at periastron. The KI $V^{2}$ data phase coverage is indicated on the visual orbit with points (they are not separation vectors); the phase coverage of the $V^{2}$ data is sparse relative to other similar analyses (e.g., Boden et al. 2000), but phase coverage provided by VLBI observations from P96 and this work complements the $V^{2}$ coverage. Further, the incorporation of the VLBI separations also breaks the $180^{\circ}-\Omega$ degeneracy inherent in $V^{2}$-only analyses (e.g., Boden et al. 2000, 2005a), so the A orbit rendered in Figure 3 is indeed as it appears on the sky. The apparent sizes of the V773 Tau A components are estimated ( $\$ 3.1$; Table 5) and rendered to scale; these same diameter values are used in the 
TABLE 3

V773 Tau A RV Data Set, "Hybrid" Model Predictions, Residuals, and Phases

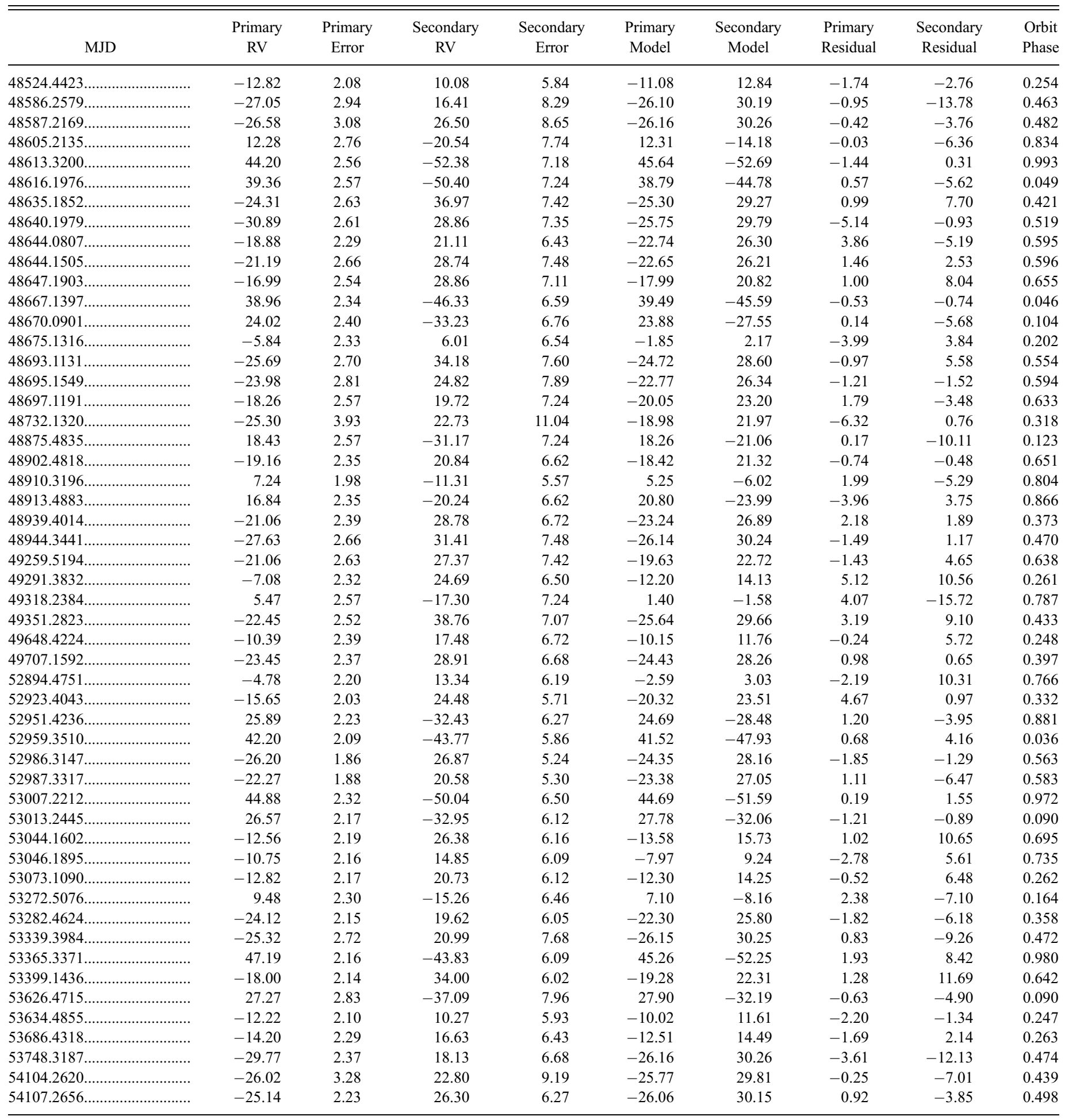

Notes.-Units of all RV quantities are in $\mathrm{km} \mathrm{s}^{-1}$. Residuals are quoted as data minus model. A model of the V773 Tau A-B orbit has been extracted from these data; details will be given in G. Torres et al. (2007, in preparation).

orbital modeling. The bottom panel depicts RV curves from the Hybrid orbit model and RV data described above.

V773 Tau A presents two significant challenges for RV orbit estimation. The first is that both components are relatively rapid rotators: W95 found the $v \sin i$ for both stars to be around $40 \mathrm{~km} \mathrm{~s}^{-1}$; this rotational broadening is seen in our spectra as well, and it significantly degrades the precision of the velocities. Second, and most notably, is the A barycenter motion that results from the A-B orbit. Figure 4 of D03 depicts the significant A-B orbital motion over the time since the detection of V773 Tau B, and our RV data set (starting in 1991) not only spans essentially all of this period but also includes the A-B periastron passage expected in 1996 (D03). It is therefore important to account for the A-B orbital motion in the modeling of the A-component RVs. 
TABLE 4

V773 Tau A Orbital Parameters

\begin{tabular}{|c|c|c|c|c|}
\hline \multirow[b]{2}{*}{ Orbital Parameter } & \multirow[b]{2}{*}{ W95 } & \multicolumn{3}{|c|}{ THIS WORK } \\
\hline & & $\mathrm{RV}$ & $V^{2}$ and $\mathrm{RV}$ & Hybrid \\
\hline Period (days) & $51.075 \pm 0.018$ & $51.1055 \pm 0.0023$ & $51.1033 \pm 0.0022$ & $51.1039 \pm 0.0021$ \\
\hline$T_{0}$ (MJD) & $49330.44 \pm 0.56$ & $53059.87 \pm 0.36$ & $53059.50 \pm 0.35$ & $53059.73 \pm 0.33$ \\
\hline e & $0.267 \pm 0.016$ & $0.275 \pm 0.012$ & $0.2708 \pm 0.0094$ & $0.2717 \pm 0.0085$ \\
\hline$K_{\mathrm{Aa}}\left(\mathrm{km} \mathrm{s}^{-1}\right)$ & $32.6 \pm 0.7$ & $35.95 \pm 0.53$ & $35.96 \pm 0.53$ & $35.90 \pm 0.53$ \\
\hline$K_{\mathrm{Ab}}\left(\mathrm{km} \mathrm{s}^{-1}\right) \ldots \ldots$ & $43.1 \pm 2.1$ & $41.6 \pm 1.4$ & $41.5 \pm 1.4$ & $41.5 \pm 1.4$ \\
\hline$\gamma\left(\mathrm{km} \mathrm{s}^{-1}\right)$ & $13.8 \pm 0.9$ & $0.00 \pm 0.31^{*}$ & $0.05 \pm 0.31^{*}$ & $0.02 \pm 0.32^{*}$ \\
\hline$\omega_{\mathrm{Aa}}(\mathrm{deg}) \ldots \ldots$ & $10.5 \pm 5.1$ & $5.1 \pm 2.6$ & $3.4 \pm 2.5$ & $4.6 \pm 2.4$ \\
\hline$\Omega(\mathrm{deg})$ & $\ldots$ & $\ldots$ & $61.5 \pm 2.5$ & $63.5 \pm 1.7$ \\
\hline$i(\mathrm{deg})$ & $\ldots$ & $\ldots$ & $65.9 \pm 2.8$ & $66.0 \pm 2.4$ \\
\hline$a$ (mas) & $\ldots$ & $\ldots$ & $2.777 \pm 0.056$ & $2.811 \pm 0.047$ \\
\hline$\Delta K(\mathrm{mag})$ & $\ldots$ & $\ldots$ & $0.559 \pm 0.082$ & $0.551 \pm 0.078$ \\
\hline
\end{tabular}

Notes.-Summarized here are orbital parameters for the V773 Tau A subsystem as determined by W95 and present results. We give three separate fits to our data sets: RV only, KI $V^{2}$ integrated with RV (" $V^{2}$ and RV"), and KI $V^{2}$, VLBI, and RV ("Hybrid"). Barycenter velocity ( $\gamma$ ) estimates (shown with an asterisk) are artificially near zero because we have estimated and removed a model of the A-B orbital motion (see discussion in text). $\Omega$ is quoted in a position angle convention.

Modeling of the A-B orbit would not have been possible at the time of W95 but becomes feasible with the present data set.

A separate RV-only orbital solution was carried out in which, in addition to solving for preliminary elements of the A orbit, we modeled the Keplerian motion of A around the A-B barycenter. In this analysis we held fixed some of the elements of the outer orbit to the values given by D03 (specifically, the period, eccentricity, longitude of periastron, and time of periastron passage), and we solved for the velocity semiamplitude of the barycenter of A $\left(K_{\mathrm{A}}=6.11 \pm 0.34 \mathrm{~km} \mathrm{~s}^{-1}\right)$. This motion was then removed from our original velocities prior to combining them with the KI and VLBI data. A more complete treatment would incorporate all the astrometric measurements for the A-B orbit (e.g., D03) along with the velocities and astrometry for the inner subsystem in a full solution solving for both orbits simultaneously. Such a study is beyond the scope of the present work but will be described in a forthcoming publication (G. Torres et al. 2007, in preparation).

There are significant differences between several of our orbital parameters for V773 Tau A and the corresponding values from
W95, most notably in the period and $K_{\mathrm{Aa}}$ (see Table 4). We attribute these differences to a combination of at least three effects: (1) limited phase coverage of the A orbit by W95, particularly near A apastron; (2) possible biases in the W95 RVs due to line blending, exacerbated by the rapid rotation of $\mathrm{Aa}$ and $\mathrm{Ab}$ along with the lower spectral resolution of that study compared to ours; and (3) residual effects from the unmodeled motion in the outer orbit in W95's solution.

P96 argue that their resolved VLBI image of V773 Tau A measures the relative component positions to within a "few" stellar diameters (the primary apparent diameter is approximately 0.15 mas; see $\S 3.1$ ). However, it is important to recognize that VLBI and KI $V^{2}$ data measure different emission mechanisms, and further, one would not expect individual radio flares to be centered on the stellar photospheres (measured by the KI $V^{2}$ data). This potential photosphere/radio offset motivated us to approach integrating the radio data into the orbit model cautiously. In our initial screening of the VLBI relative astrometry we compared the radio observations with position predictions from a $V^{2}$ and RV orbit model (Table 4). We found that the model

TABLE 5

V773 Tau A Physical Parameters

\begin{tabular}{|c|c|c|}
\hline Physical Parameter & $\begin{array}{l}\text { Primary } \\
\text { (Aa) }\end{array}$ & $\begin{array}{l}\text { Secondary } \\
\text { (Ab) }\end{array}$ \\
\hline$a(\mathrm{AU}) \ldots$ & $0.1777 \pm 0.0042$ & $0.2053 \pm 0.0070$ \\
\hline Mass $\left(M_{\odot}\right)$ & $1.54 \pm 0.14$ & $1.332 \pm 0.097$ \\
\hline System mass $\left(M_{\odot}\right)$ & \multicolumn{2}{|c|}{$2.87 \pm 0.18$} \\
\hline System distance $(\mathrm{pc})$ & \multicolumn{2}{|c|}{$136.2 \pm 3.7$} \\
\hline$\pi_{\text {orb }}(\mathrm{mas})$ & \multicolumn{2}{|c|}{$7.34 \pm 0.20$} \\
\hline$T_{\text {eff }}(\mathrm{K})$ & $4900 \pm 150$ & $4740 \pm 200$ \\
\hline Model diameter (mas) & $0.152 \pm 0.014$ & $0.119 \pm 0.011$ \\
\hline Bolometric flux $\left(10^{-9} \mathrm{ergs} \mathrm{cm}^{-2} \mathrm{~s}^{-1}\right) \ldots \ldots \ldots \ldots \ldots \ldots \ldots$ & $4.44 \pm 0.40$ & $2.38 \pm 0.15$ \\
\hline 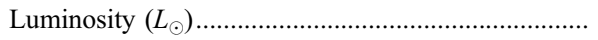 & $2.56 \pm 0.35$ & $1.37 \pm 0.15$ \\
\hline Radius $\left(R_{\odot}\right)$ & $2.22 \pm 0.20$ & $1.74 \pm 0.19$ \\
\hline $\log g$ & $3.930 \pm 0.094$ & $4.081 \pm 0.088$ \\
\hline$M_{K}(\mathrm{mag})$ & $1.75 \pm 0.12$ & $2.30 \pm 0.13$ \\
\hline$M_{V}(\mathrm{mag})$ & $3.90 \pm 0.16$ & $4.98 \pm 0.16$ \\
\hline$V-K(\mathrm{mag})$ & $2.15 \pm 0.14$ & $2.68 \pm 0.14$ \\
\hline
\end{tabular}

Note.-Summarized here are physical (and related) parameters for the V773 Tau A subsystem as derived primarily from the "Hybrid" orbit solution (Table 4) and radiometric modeling (§ 3.1). 
reasonably reproduced the observed radio separations with a typical residual of $0.2-0.3$ mas $\left(\approx 4 R_{*}\right)$, slightly larger than the formal errors expected from the VLBI synthesized beam and the $\mathrm{S} / \mathrm{N}$ in the image, but in good agreement with the expected IR/radio offsets. The agreement we see between the radio separations and the $V^{2}$ and RV orbit model makes it clear that the VLBI data do contain useful information on the component separations. We therefore felt confident in integrating the KI $V^{2}$ and four VLBI observations (including the P96 epoch) into our orbit model estimation. An analysis of the radio morphologies seen in the VLBA+EB observations will be presented in a future publication (Massi et al. 2007).

Figure 1 depicts direct comparisons between our KI $V^{2}$ observations and predictions from our V773 Tau A Hybrid orbit model from Table 4 (the five nights of data are each rendered in separate subpanels). The model is seen to be in good agreement with the KI data. Further, Figure 3 shows a direct comparison between the VLBA separations and the Hybrid visual orbit model. Again, the body of the VLBI data is in good agreement with the orbit model, and the VLBI phase coverage complements the phase coverage provided by the KI $V^{2}$ data.

Orbital parameter estimates for the V773 Tau A subsystem are summarized in Table 4. Included for relative comparison are the orbital parameters determined by W95, our RV double-lined orbit ("RV"), the KI $V^{2}$ and RV solution (" $V 2$ and RV"), and the full integrated solution including the RV, $V^{2}$, and VLBA data ("Hybrid"). The Hybrid designation for the composite orbit model recognizes its unique inclusion of both near-IR and radio interferometry data; this model is our favored orbit solution and is used in the remaining analysis. Again note that the A barycenter velocity is artificially near zero in our orbital solutions as the RV measurements have been corrected for our model of the A-B orbital motion.

\section{V773 TAU A PHYSICAL PROPERTIES}

The orbital parameters from Table 4 allow us to directly compute many of the physical properties of the V773 Tau A subsystem and its components. Physical parameters derived from our Hybrid integrated visual/spectroscopic orbit are summarized in Table 5. The dynamical masses resulting from our orbit model for the two A components are $1.54 \pm 0.14$ and $1.332 \pm 0.097 M_{\odot}$ for $\mathrm{Aa}$ and $\mathrm{Ab}$, respectively. This primary mass is in acceptable agreement with inferences by White \& Ghez (2001, hereafter WG01) and Palla \& Stahler (2001) based on radiometric properties (i.e., luminosity and effective temperature). Because it bears on the A-B orbit analysis (G. Torres et al. 2007, in preparation), we estimate the A subsystem mass as $2.87 \pm 0.18 M_{\odot}$.

L99 estimated a V773 Tau system distance of $148.4 \pm 5.5 \mathrm{pc}$. The distance determination to V773 Tau A derived from our visual and physical orbital solution is $136.2 \pm 3.7 \mathrm{pc}$, corresponding to an orbital parallax of $7.34 \pm 0.20$ mas, and consistent with the L99 result at $8.1 \%$ and $1.9 \sigma$. L99 describe their distance estimate error as "formal," and it apparently did not account for the orbital motion of A in their data reduction. With these caveats we believe that the L99 distance estimate and ours are in acceptable agreement.

At the distance of V773 Tau, neither of the A subsystem components is significantly resolved by the KI $K$-band fringe spacing, and we must resort to model diameters for the components. We have estimated the V773 Tau A component apparent diameters through SED modeling; details of the SED modeling are given in $\S 3.1$. We find apparent diameters of $0.152 \pm 0.014$ and $0.119 \pm 0.011$ mas for the $\mathrm{Aa}$ and $\mathrm{Ab}$ components, respectively. With our system distance estimate these estimated diameters cor-
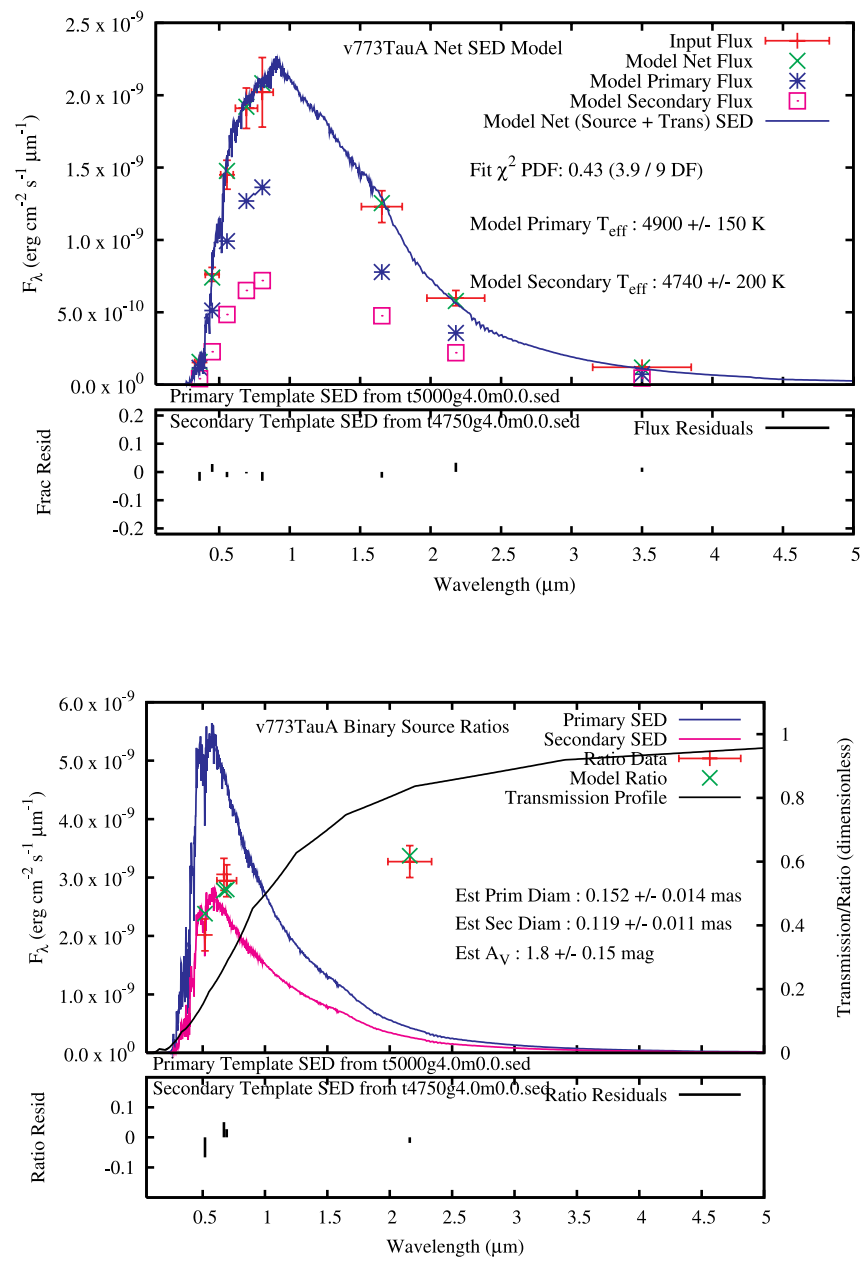

FIG. 4.-Sample SED model for V773 Tau A. Here SED templates from Lejeune et al. $(1997,1998)$ have been used to simultaneously model published flux measurements (from WG01 and D03) and flux ratio estimates (Table 4; bottom) from our KI $V^{2}$ and spectroscopic measurements.

respond to physical radii of $2.22 \pm 0.20$ and $1.74 \pm 0.19 R_{\odot}$ and (combined with the mass estimates) log surface gravities of $3.930 \pm 0.094$ and $4.081 \pm 0.088$ for the Aa and Ab components, respectively. Similarly, we have estimated absolute magnitudes in $V$ and $K$ and a $V-K$ color index for each of the components (Table 5), but these are of limited precision as they are dominated by the uncertainty in the extinction to the system $(\S 3.1)$.

\subsection{Spectral Energy Distribution Modeling}

Because interferometric observations potentially resolve the stellar components in a binary system, we always construct SED models for binary systems to estimate a priori apparent diameters. Sample results of our SED modeling of V773 Tau A are shown in Figure 4. Our modeling is based on visible and near-IR photometry on A from WG01 and D03, respectively (summarized in Table 6), and component flux ratios from W95 and our visible spectroscopy and near-IR KI $V^{2}$ data (discussed above). Using a custom two-component SED modeling code, we have modeled the A subsystem flux and ratios using solar abundance, intermediate surface gravity SED templates from Kurucz mod$\mathrm{els}^{9}$ and Lejeune et al. (1997, 1998). We model the flux and ratio data with a large grid of SED templates for each component

\footnotetext{
${ }^{9}$ Kurucz models used here are available at http://cfaku5.cfa.harvard.edu.
} 
TABLE 6

V773 TAU A Photometry

\begin{tabular}{|c|c|c|}
\hline Passband & Magnitude & References \\
\hline Johnson $U \ldots .$. & $13.60 \pm 0.12$ & WG01 \\
\hline Johnson $B \ldots$. & $12.31 \pm 0.07$ & WG01 \\
\hline Johnson $V \ldots \ldots$ & $11.03 \pm 0.08$ & WG01 \\
\hline 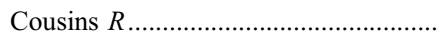 & $10.13 \pm 0.08$ & WG01 \\
\hline 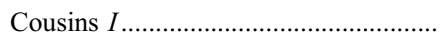 & $9.34 \pm 0.14$ & WG01 \\
\hline Johnson $H \ldots .$. & $7.43 \pm 0.1$ & D03 \\
\hline 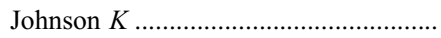 & $7.10 \pm 0.1$ & D03 \\
\hline Johnson $L$ & $6.85 \pm 0.1$ & D03 \\
\hline
\end{tabular}

NoтE.-Visible photometry from WG01; near-IR photometry from D03.

ranging in effective temperature (spectral type) from 4250 to $5500 \mathrm{~K}$, surface gravity of $\log g=4.0$, and including extinction as a free variable in the parameter estimation. Constrained by the ratio data, this SED modeling preferred component temperature differences over a reasonably narrow range $\left(\Delta T_{\text {eff }} \sim 150-250 \mathrm{~K}\right)$, consistent with our spectroscopic study. But because the component colors are somewhat degenerate with reddening from dust extinction, we found acceptable solutions over a relatively broad range $(\sim 500 \mathrm{~K})$ of component temperatures. We have therefore adopted templates corresponding to the spectroscopic temperatures (4900 and $4740 \mathrm{~K}$ for $\mathrm{Aa}$ and $\mathrm{Ab}$, respectively). These temperatures acceptably agree with the composite temperature estimated by WG01, but the hotter primary in our modeling prefers a slightly higher extinction value $\left(A_{V}=1.80 \pm 0.15\right.$ vs. $1.39 \pm$ 0.17 from WG01). The resulting component bolometric fluxes and luminosities (the second based on our distance determination) are summarized in Table 5. In particular, we find luminosities of $2.56 \pm 0.35$ and $1.37 \pm 0.15 L_{\odot}$ for $\mathrm{Aa}$ and $\mathrm{Ab}$, respectively; these values are in good agreement with the range of previous component luminosity estimates from W95, Ghez et al. (1997), and D03.

\section{DISCUSSION}

\subsection{Comparison with Stellar Evolution Models}

Hillenbrand \& White (2004) and Mathieu et al. (2007) have recently summarized comparisons between PMS stellar models and PMS stars with dynamical mass determinations. In particular, Hillenbrand \& White (2004) have shown that PMS models are in good agreement with dynamically determined masses above 1.2 $M_{\odot}$. However, below $1.2 M_{\odot}$ the existing models do a poorer job of matching observed component properties, tending to predict hotter and more luminous stars for a given mass.

Figure 5 presents theoretical H-R diagram (luminosity $/ T_{\text {eff }}$ ) comparisons between our determinations of V773 Tau-A component radiometric properties and solar composition PMS models from Siess et al. (2000), Yonsei-Yale (Demarque et al. 2004; herein $\mathrm{Y}^{2}$ ), D'Antona \& Mazzitelli (1997), and Palla \& Stahler (1999). (Popular models by Baraffe et al. [1998] were excluded only because they do not cover the mass range for these stars.) These models span a wide range of physical ingredients and assumptions, some more realistic than others for the particular mass range of interest. In these figures evolutionary tracks at fixed mass between 1.2 and $1.8 M_{\odot}$ (in steps of $0.1 M_{\odot}$ as available from the relevant models) are shown in solid lines, with thick lines indicating tracks at masses close to dynamical masses from Table 5. Superimposed in dotted lines are isochrones at a relevant range of ages $(1,3,5,7$, and $10 \mathrm{Myr}$ as available from the relevant models). Finally, the $\mathrm{Y}^{2}$ models support comput- ing tracks at user-defined masses; tracks at the estimated component masses from Table 5 are shown for Aa $\left(1.54 M_{\odot} ;\right.$ blue $)$ and $\mathrm{Ab}\left(1.332 M_{\odot} ;\right.$ red $)$.

As expected from Hillenbrand \& White (2004), all the surveyed PMS models are in reasonable (e.g., $2 \sigma$ ) agreement for the V773 Tau A component mass range. The models indicate that the V773 Tau A components are still in their quasi-isothermal ("Hayashi") contraction phase. More quantitatively some model predictions seem to match the A-component properties better than others. For instance, the solar abundance Siess et al. (2000) (top left) and Palla \& Stahler (1999) (bottom right) tracks near the dynamical masses tend to underpredict our estimated component temperatures and luminosities. In particular, for the Siess et al. (2000) models, this is similar to our previous results for HD 98800B (Boden et al. 2005b). This apparent temperature discrepancy seems to reinforce a suggestion by Montalbán et al. (2004) that the solar abundance Siess et al. (2000) models appear to be systematically cool compared with other models. Conversely, the D'Antona \& Mazzitelli (1997) tracks (bottom left) at our mass values appear to slightly overpredict our component temperatures and luminosity estimates. The $\mathrm{Y}^{2}$ model predictions (top right; including tracks for the specific masses of the components) are seen to match our parameter determinations very well.

Figure 6 gives a final model comparison. Recently, Montalbán \& D'Antona (2006) have updated D'Antona \& Mazzitelli (1997) models with a revised "2D radiative hydrodynamic" (2D RHD) convection treatment. Figure 6 shows the result of this updated modeling with tracks from D'Antona \& Mazzitelli (1997) in black and from Montalbán \& D'Antona (2006) in blue; the presentation is similar to that given in Figure 5, except we have displayed only mass tracks near the component dynamical mass estimates $\left(1.3\right.$ and $\left.1.5 M_{\odot}\right)$. The $2 \mathrm{D}$ RHD convective treatment increases the convective "efficiency" and energy transport in the atmosphere, moving the tracks toward lower temperatures during the Hayashi phase, and brings the model predictions into excellent agreement with the component parameters presented here.

Isochrones from the models we considered would predict a range of component ages from 2 to $7 \mathrm{Myr}$, but restricting attention to the Montalbán \& D'Antona (2006) and $\mathrm{Y}^{2}$ models, a more plausible age estimate is $3 \pm 1 \mathrm{Myr}$, with both components appearing coeval to within present uncertainties. Such a system age is broadly consistent with age estimates for the Taurus population using a variety of evolutionary models (e.g., WG01; Palla \& Stahler 2002; Luhman 2004). More specifically, this age estimate would put V773 Tau A in the putative intermediate-age population for Taurus as defined by Palla \& Stahler (2002).

\subsection{Summary and Conclusions}

We have presented new interferometric (Tables 1 and 2) and RV data (Table 3) on the PMS binary subsystem V773 Tau A. Modeling these data in conjunction with older VLBI astrometry from $\mathrm{P} 96$ allows us to estimate the visual and physical orbits for V773 Tau A, as well as physical parameters of the components, and place significant constraints on PMS stellar evolution models. We characterize the V773 Tau A orbit model presented here as preliminary; we plan additional RV, VLBA, and KI observations that will further refine the physical orbit model for the A subsystem and constrain the component parameters. Further, ongoing RV and astrometric observations will continue also in order to probe the A-B orbit, and this in turn will refine the A subsystem RVs and orbit. Details of A-B orbit modeling will be documented in a future publication (G. Torres et al. 2007, in preparation). 

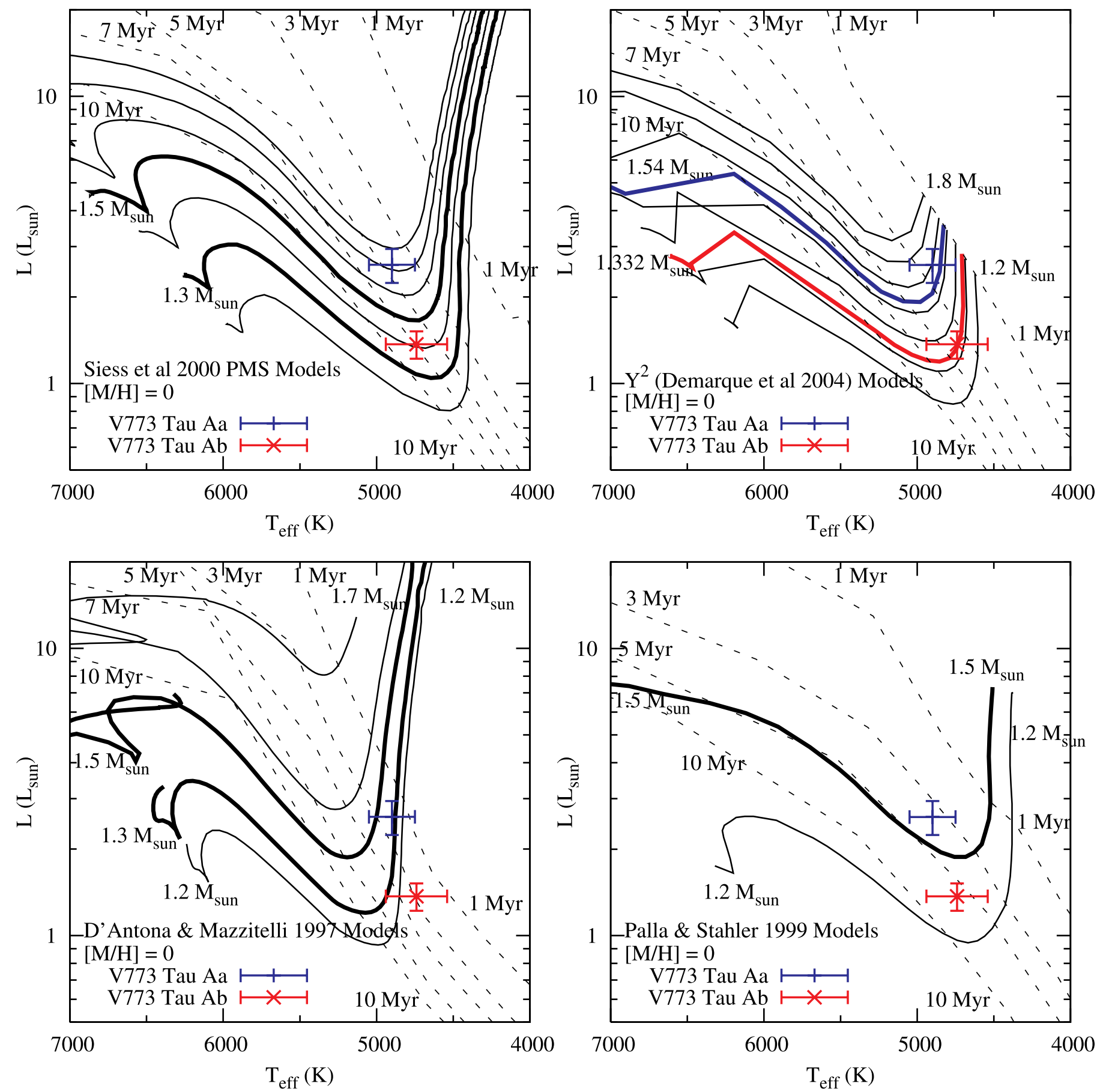

FIG. 5.-V773 Tau A components compared with PMS models. Here we show the V773 Tau A components in theoretical H-R diagrams (luminosity/ $\left.T_{\text {eff }}\right)$ and solar composition PMS evolutionary tracks and isochrones by Siess et al. (2000) (top left), Y ${ }^{2}$ (Demarque et al. 2004) (top right), D'Antona \& Mazzitelli (1997) (bottom left),

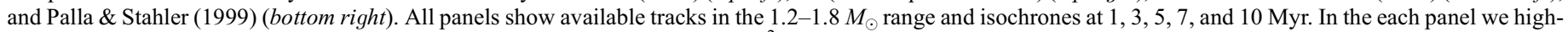

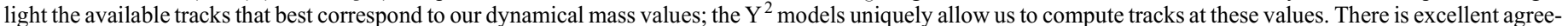

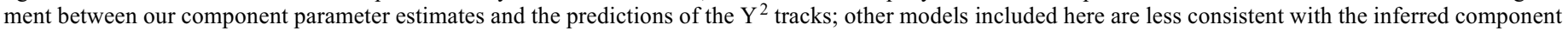
parameters.

The V773 Tau physical parameters inferred from our orbit model are in good agreement with previous results. The most notable difference in our results with previous work is in the system distance; our distance estimate is at modest variance with the VLBA parallax from L99. However, the L99 data reduction did not account for the binarity of $\mathrm{A}$, which is clearly resolved in multiple epochs (P96; this work). With that caveat we believe that the L99 distance and ours are in acceptable agreement. Because our distance estimate depends only on the appar- ent and physical orbits, we believe that it is likely accurate at its stated uncertainty. We estimate our relative component mass errors at $9.1 \%$ and $7.3 \%$ for $\mathrm{Aa}$ and $\mathrm{Ab}$, respectively, making them comparable with other PMS stellar dynamical mass determinations (Hillenbrand \& White 2004; Mathieu et al. 2007).

It is noteworthy that the P96 and present VLBI data agree well with the A-orbit model presented here (including a model that excludes the VLBI data themselves; see, e.g., Fig. 2). Narrowly this would seem to support the P96 interpretation that both A 


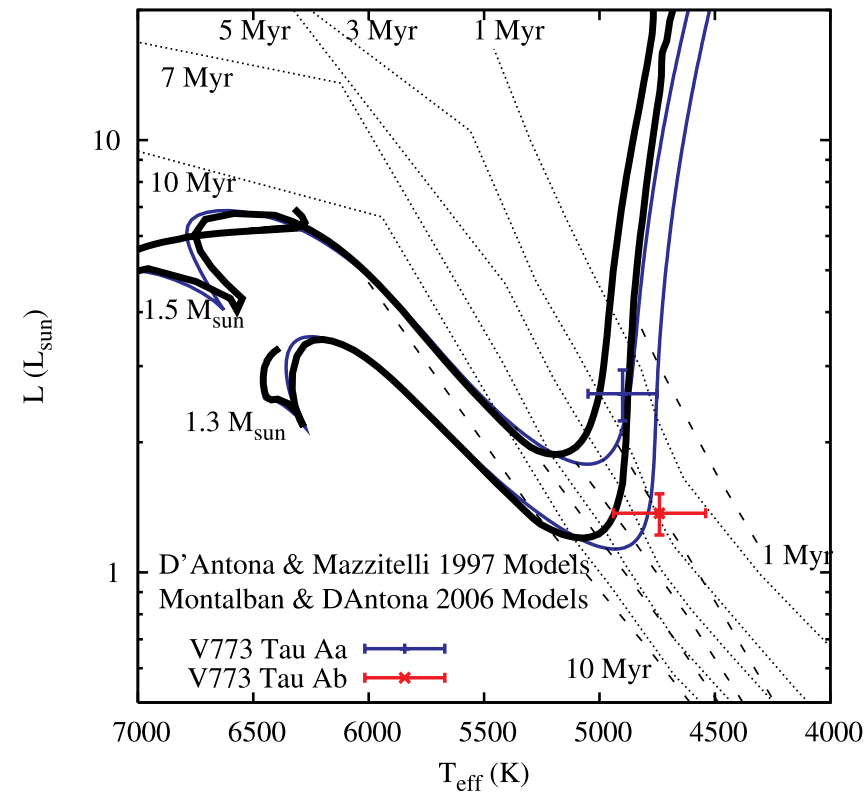

FIG. 6.-V773 Tau A components compared with PMS models from D'Antona \& Mazzitelli (1997) and Montalbán \& D’Antona (2006). The presentation is similar to Fig. 5, but here we focus on tracks near the A-component dynamical masses (1.3 and 1.5 $\left.M_{\odot}\right)$. The Montalbán \& D'Antona (2006; tracks in blue, isochrones in thick dotted lines) models updated the older D'Antona \& Mazzitelli (1997; tracks in black, isochrones in thin dotted lines) models with a revised convection model, bringing the updated models into excellent agreement with the mass and radiometric parameters estimated here.

components are active radio emitters. More broadly, the ability to compare the radio morphology with at-epoch estimates of the component positions would seem to present an opportunity to constrain the radio emission and magnetic field topology in these stars. A more complete analysis of the VLBI data in this context will be made in a forthcoming publication (Massi et al. 2007).

V773 Tau A radiometric estimates must necessarily account for (and are complicated by) extinction. Our estimate of the extinction is in reasonable agreement with earlier work (e.g., W95; Ghez et al. 1997; WG01) but prefers a slightly higher extinction when constrained by the spectroscopic temperatures (which are quasi-independent of the extinction). With this uncertainty in mind, we have made relatively conservative estimates of the errors in the radiometric properties.

Even with the relatively conservative error estimates, the combination of our component dynamical mass and radiometric parameter estimates appears to differentiate between the range of solar abundance PMS models considered here. In particular, we find better agreement between the estimated component parameters and predictions of solar abundance models from $Y^{2}$ and Montalbán \& D'Antona (2006) than other models considered here, and these two models agree on a V773 Tau age estimate of approximately $3 \pm 1 \mathrm{Myr}$; this is in good agreement with the multiple epochs of Taurus star formation (Palla $\&$ Stahler 2002). This age would place V773 Tau in the second of three age groups defined by Palla \& Stahler (2002), i.e., stars with age $2 \mathrm{Myr}<t<4 \mathrm{Myr}$. Such and younger stars are typically concentrated along the "central filaments" of the Taurus molecular cloud systems. V773 Tau appears in fact located at the periphery of a $\sim 0.7 \times 0.5 \mathrm{pc}-$-sized "core" imaged in the high column density tracing $\mathrm{C}^{18} \mathrm{O}$ molecule by Onishi et al. (1996, 1998; their core 5). From the observed intensity of the $\mathrm{C}^{18} \mathrm{O} J=1-0$ line one can derive an $\mathrm{H}_{2}$ column density that translates into an $A_{V}>5$. Extinction maps from Digitized Sky Survey source counts around V773 Tau's position also indicate an $A_{V}$ around 5 but have a "resolution" of several arcminutes (Dobashi et al. 2005). These extinction values are several times larger than our and other extinction determinations for V773 Tau A (§ 3.1) and seem to indicate that the system is located just at the near border of a denser cloud core.

Relative to the component parameters estimated here, the Siess et al. (2000) and Palla \& Stahler (1999) models predict cooler and less luminous components, while the D'Antona \& Mazzitelli (1997) models predict hotter and more luminous components. However, these conclusions would change if the component mass estimates were to systematically change (i.e., both components either more or less massive than present estimates) by only about $2 \sigma$. Qualitatively this seems relatively unlikely, but given the uncertainties in this study and the added complexity of multiple orbits in the V773 Tau system, it would be prudent to withhold final judgment until the modeling of the A-B orbit improves with time and there is an expanded astrometric and RV data set.

Finally, it is important to note that placing constraints on stellar evolutionary models in general, and PMS evolutionary models in particular, requires precise measurement of more than just component dynamical mass, but also equally important parameters such as luminosity, $T_{\text {eff }}$, and abundance. Even at the present level of the work here (i.e., dynamical masses better than $10 \%, 1 \sigma$ ), uncertainties in $L$ and $T_{\text {eff }}$ are significant factors in limiting conclusions we can draw about model predictions. As for abundance, we have restricted our comparisons of V773 Tau A component properties with solar composition models. Fundamentally this is a practical consideration; some of the model sets considered here only support solar abundances. Nominally abundances in Taurus-Auriga (and by consequence V773 Tau) are thought to be near solar values (see Padgett 1996). However, as in other sectors of the H-R diagram, more secure abundance determinations in star-forming regions, along with improved measurements of luminosity and temperature, will be critical for definitive tests of PMS models.

Part of this work was performed at the Michelson Science Center (MSC), California Institute of Technology under contract with the National Aeronautics and Space Administration (NASA).

Some of the data presented herein were obtained at the W. M. Keck Observatory, which is operated as a scientific partnership among the California Institute of Technology, the University of California, and NASA. The Observatory was made possible by the generous financial support of the W. M. Keck Foundation. We gratefully acknowledge the support of personnel at the Jet Propulsion Laboratory, W. M. Keck Observatory, and the MSC in obtaining KI observations of V773 Tau A. The authors wish to recognize and acknowledge the very significant cultural role and reverence that the summit of Mauna Kea has always had within the indigenous Hawaiian community. We are most fortunate to have the opportunity to conduct observations from this mountain.

The CfA RV observations presented here were originally advocated by Robert Mathieu; we thank him for his gracious contribution of these data in their use here. We thank P. Berlind, M. Calkins, J. Caruso, R. J. Davis, G. Esquerdo, J. Peters, A. Milone, and R. P. Stefanik for obtaining many of the spectroscopic observations. G. T. acknowledges partial support from NASA's MASSIF SIM Key Project (BLF57-04) and NSF grant AST 04-06183. 
We thank F. D'Antona, J. Montalbán, S. Stahler, and F. Palla for sharing their pre-main-sequence evolutionary models and for fruitful discussions on applying them. Thanks also to G. Duchene for sharing IR photometry for V773 Tau A (including his kind permission to report these data here in Table 6) and the anonymous referee, whose many thoughtful comments helped greatly improve this manuscript.
This research has made use of services of the MSC at the California Institute of Technology; the SIMBAD database, operated at CDS, Strasbourg, France; NASA's Astrophysics Data System Abstract Service; and data products from the Two Micron All Sky Survey, which is a joint project of the University of Massachusetts and the Infrared Processing and Analysis Center, funded by NASA and the National Science Foundation.
Baraffe, I., et al. 1998, A\&A, 337, 403

Boden, A., Torres, G., \& Hummel, C. 2005a, ApJ, 627, 464

Boden, A., et al. 2000, ApJ, 536, 880 2005b, ApJ, 635, 442

Colavita, M. 1999, PASP, 111, 111

Colavita, M., et al. 2003, ApJ, 592, L83

D’Antona, F., \& Mazzitelli, I. 1997, Mem. Soc. Astron. Italiana, 68, 807

Demarque, P., et al. 2004, ApJS, 155, 667

Dobashi, K., et al. 2005, PASJ, 57, 417

Duchene, G., et al. 2003, ApJ, 592, 288 (D03)

Feigelson, E., et al. 1994, ApJ, 432, 373

Ghez, A., Neugebauer, G., \& Matthews, K. 1993, AJ, 106, 2005

Ghez, A., White, R., \& Simon, M. 1997, ApJ, 490, 353

Hartigan, P., Strom, K., \& Strom, S. 1994, ApJ, 427, 961

Hillenbrand, L., \& White, R. 2004, ApJ, 604, 741

Kutner, M., Rydgren, A., \& Vrba, F. 1986, AJ, 92, 895

Latham, D. W. 1992, in IAU Colloq. 135, Complementary Approaches to Double and Multiple Star Research, ed. H. A. McAlister \& W. I. Hartkopf (ASP Conf. Ser. 32; San Francisco: ASP), 110

Latham, D. W., et al. 2002, AJ, 124, 1144

Leinert, C., et al. 1993, A\&A, 278, 129

Lejeune, T., et al. 1997, A\&AS, 125, 229 1998, A\&AS, 130, 65

Lestrade, J.-F., et al. 1999, A\&A, 344, 1014 (L99)

Luhman, K. 2004, ApJ, 617, 1216

Martín, E., et al. 1994, A\&A, 282, 503

Mason, B., et al. 2001, AJ, 122, 3466

Massi, M., Menten, M., \& Neidhofer, J. 2002, A\&A, 382, 152

Massi, M., et al. 2006, A\&A, 453, 959 2007, A\&A, submitted

\section{REFERENCES}

Mathieu, R., Baraffe, I., Simon, M., Stassun, K. G., \& White, R. 2007, in Protostars and Planets V, ed. B. Reipurth, D. Jewitt, \& K. Keil (Tucson: Univ. Arizona Press), 411

Montalbán, J., \& D’Antona, F. 2006, MNRAS, 370, 1823

Montalbán, J., et al. 2004, A\&A, 416, 1081

Napier, P., et al. 1994, Proc. IEEE, 82, 658

Nordström, B., et al. 1994, A\&A, 287, 338

O’Neal, D., et al. 1990, AJ, 100, 1610

Onishi, T., et al. 1996, ApJ, 465, 815 1998, ApJ, 502, 296

Padgett, D. 1996, ApJ, 471, 847

Palla, F., \& Stahler, S. 1999, ApJ, 525, 772 2001, ApJ, 553, 299 2002, ApJ, 581, 1194

Phillips, R., et al. 1996, AJ, 111, 918 (P96)

Rydgren, A., Strom, S., \& Strom, K. 1976, ApJS, 30, 307

Siess, L., Dufour, E., \& Forestini, M. 2000, A\&A, 358, 593

Stefanik, R. P., Latham, D. W., \& Torres, G. 1999, in IAU Colloq. 170, Precise Stellar Radial Velocities, ed. J. B. Hearnshaw \& C. D. Scarfe (ASP Conf. Ser. 185; San Francisco: ASP), 354

Tamazian, V., et al. 2002, ApJ, 578, 925

Torres, G., et al. 2003, AJ, 125, 825

Welty, A. 1995, AJ, 110, 776 (W95)

White, R., \& Ghez, A. 2001, ApJ, 556, 265 (WG01)

Woitas, J. 2003, A\&A, 406, 685

Zensus, J., Diamond, P., \& Napier, P., eds. 1995, ASP Conf. Ser. 82, Very Long

Baseline Interferometry and the VLBA (San Francisco: ASP)

Zucker, S., \& Mazeh, T. 1994, ApJ, 420, 806

Zucker, S., Torres, G., \& Mazeh, T. 1995, ApJ, 452, 863 